\title{
Diferencias de pesos entre terneros Braford enteros y castrados a diferentes edades
}

\author{
Cappello, J.S. ${ }^{1}$; Bernardis, M.C. ${ }^{2}$; Juarez, J.O. ${ }^{2}$; Capellari, A. ${ }^{1}$ \\ ${ }^{1}$ Docentes Facultad de Ciencias Veterinarias UNNE, Cabral 2139, \\ Corrientes 3400 (Argentina). ${ }^{2}$ Veterinarios actividad privada \\ (Tartagal, Santa Fe).E-mail: scappello@vet.unne.edu.ar
}

\begin{abstract}
Resumen
Cappello, J.S.; Bernardis, M.C.; Juarez, J.O.; Capellari, A.: Diferencias de pesos entre terneros Braford enteros y castrados a diferentes edades. Rev. Vet. 31 1, 50-53, 2020. Se compararon terneros braford machos enteros y castrados, con diferentes edades de esterilización, con el objetivo de evaluar este efecto en el crecimiento mediante la ganancia total y diaria de peso. Se utilizaron 28 terneros al pie de madre en campo natural. Se dividieron aleatoriamente en grupos de 7 individuos: C45: castrados a los 45 días de edad; E45: enteros con 45 días de edad; C90: castrados a los 90 días de edad y E90: enteros con 90 días de edad. Los pesos se registraron individualmente el día 0 (día de la castración) y posteriormente los días 15,30 y 45 . La técnica de castración se realizó por cirugía cruenta. Las variables estudiadas para el peso vivo fueron: ganancia total (GT, $\mathrm{kg}$ ) y ganancia media diaria (GMD, $\mathrm{kg}$ / día). Se obtuvieron estadísticas descriptivas, análisis de varianza (ANOVA) y test de Duncan $(\mathrm{p}<0,05)$, tomándose como efecto principal la edad de castración (45 y 90 días), obrando los enteros como testigos. Las medias para GT fueron: C45: 34,86 kg; E45: 37,29 kg; C90: 51,14 $\mathrm{kg}$ y E90: 44,29 kg. Para GMD fueron: C45: 0,58 kg; E45: 0,62 kg; C90: 0,85 kg y E90: 0,74 $\mathrm{kg}$. El ANOVA arrojó diferencias significativas para ambas variables: $\mathrm{GT}(\mathrm{p}=0,0156)$ y GMD $(\mathrm{p}=0,0157)$, siendo C90 superior al resto de los grupos. Por su parte, E90 obtuvo valores intermedios entre C90 y los terneros de 45 días. Entre C45 y E45 no se hallaron diferencias significativas en ambas variables. Se concluye que el momento elegido para la castración debe ser cercano a los tres meses, ya que a los 45 días el peso vivo resultó más afectado por la cirugía.
\end{abstract}

Palabras clave: terneros, castración, crecimiento, ganancia diaria de peso.

\begin{abstract}
Cappello, J.S.; Bernardis, M.C.; Juarez, J.O.; Capellari, A.: Evaluation of growth of intact vs castrated Braford calves at different ages. Rev. Vet. 31 1, 50-53, 2020. We compared entire versus castrated male Braford calves, at different ages of castration, with the objective to evaluate this effect on growth by means of the total and daily weight gain. Twenty-eight Braford male suckling calves, were used. Animals were randomly divided into groups of 7 individuals: C45: castrated at 45 days of age; E45: entire, with 45 days of age; C90: castrated at 90 days of age and E90: entire, with 90 days of age. Individual weights were recorded on day 0 (castration's day) and then on days 15, 30 and 45. Castration was performed by means of surgery. The studied variables were total gain (GT, kg of live weight) and average daily gain (GMD, kg/day). Descriptive statistics, analysis of variance (ANOVA) and Duncan's test $(\mathrm{p}<0.05)$ were performed, considering as the main effect the age of castration ( 45 and 90 days), with the intact males as controls. The means for GT were: C45: $34.86 \mathrm{~kg}$; E45: 37.29 kg; C90: $51.14 \mathrm{~kg}$ and E90: $44.29 \mathrm{~kg}$. For GMD, they were: C45: $0.58 \mathrm{~kg}$; E45: $0.62 \mathrm{~kg}$; C90: $0.85 \mathrm{~kg}$ and E90: $0.74 \mathrm{~kg}$. The ANOVA test showed significant differences for both variables, GT ( $\mathrm{p}=0.0156)$ and GMD ( $\mathrm{p}=0.0157)$, with $\mathrm{C} 90$ being the higher, considering the other groups. In particular, E90 group obtained intermediate values between C90 and calves of 45 days. Between C45 and E45, no significant differences were found for both variables. We conclude that a recommended castration time should be close to three months of age, since at 45 days of age live weight was more affected by the surgical technique.
\end{abstract}

Key words: calves, castration, growth, daily weight gain. 


\section{INTRODUCCIÓN}

El nordeste argentino abarca una superficie mayor a 30.000 .000 de ha. Su clima es subtropical húmedo, con un promedio anual de precipitaciones que se incrementa desde $900 \mathrm{~mm}$ en el margen oeste de la región, hasta $1.800 \mathrm{~mm}$ en su extremo oriental. La temperatura media disminuye de norte a sur, $\operatorname{con} 23^{\circ} \mathrm{C}$ en Formosa y $19^{\circ} \mathrm{C}$ en E.Ríos ${ }^{17}$.

En el norte de Santa Fe, zona donde se realizó el estudio, el índice para clasificar los sistemas de producción de carne, cuya relación es: (novillos + novillitos / vacas), determina la orientación productiva en ganadería, siendo de 0,51 (sistemas de invernada más cría), resultando ser de ciclo completo más invernada de compra, con un porcentaje de destete del $58 \%{ }^{17}$.

Los sistemas de producción extensivos están posicionados favorablemente en varios aspectos relativos al bienestar de los animales. Sin embargo, existen factores que amenazan a estas condiciones de realización, entre las que se destacan algunas prácticas tradicionales comunes en el rodeo de cría (descorne, castración, marcación, señalada, vacunaciones y destete), así como el manejo en general del trato a los animales (agresividad, violencia innecesaria y mal uso de los caninos) ${ }^{7}$.

Lograr mayores ganancias diarias de peso vivo en los terneros redundaría en una mayor eficiencia en el sistema productivo ${ }^{4}$, por lo cual asume importancia el trato que reciben y la disminución de las causas de estrés a las que sean expuestos. Una de las prácticas a las que son sometidos los machos es la castración, la cual es realizada junto con el destete, una de las medidas de manejo más estresantes ${ }^{3,8}$.

El propósito de la esterilización es facilitar las tareas con la hacienda al reducir la agresividad y el comportamiento sexual, contribuyendo a alcanzar el peso de faena más rápido. Adicionalmente, provee una mejor calidad de res con un alto porcentaje de marmoleo y mayor espesor de grasa subcutánea, siendo ello deseado por las plantas frigoríficas, ya que la res en cámara se protege de los efectos del frío, evitando ocasionando las carnes oscuras o con aspectos visuales despreciables ${ }^{13,15,18}$.

No obstante, si se realiza antes de la pubertad, posee un efecto negativo sobre el desarrollo, el cual disminuye la ganancia diaria de peso por una menor síntesis de testosterona ${ }^{14}$. La castración tradicional de los terneros en Argentina se realiza entre los 6 y 8 meses de edad, en meses fríos y a testículo abierto con bisturí, sin uso de anestésicos ni productos mitigantes del dolor ${ }^{7,11,12}$.

Existen diferentes procedimientos, cruentos e incruentos. Los primeros, a través del uso de cuchillo o bisturí, y -entre los segundos- los anillos de goma y la pinza de Burdizzo (emasculadota). La información científica sobre los métodos de esterilización en los terneros y el bienestar animal es diversa y en algunos casos contradictoria ${ }^{7,11,12}$.

Cuando la extirpación de los testículos se realiza al nacimiento, las pérdidas ponderales son mínimas, incrementando cuadráticamente en la medida que aumenta la edad en la que se realiza la práctica ${ }^{2}$.

Diferentes organismos internacionales sugieren la ejecución de la castración al momento del nacimiento o poco después del mismo ${ }^{5} \mathrm{y}$ antes de los 2 o 3 meses de vida ${ }^{10}$. La eliminación testicular a edades tempranas permite obtener mayor marmoleo que aquellas que se esterilizan más tarde. Asimismo, los animales castrados a menor edad tienen mayor potencial para aumentar la terneza de la carne, debido a que su síntesis de testosterona es menor ${ }^{14}$.

Acorde a lo mencionado, el presente trabajo tiene como objetivo evaluar mediante la ganancia total y diaria de peso, el crecimiento de terneros Braford (machos enteros y castrados), con dos edades de esterilización (45 y 90 días).

\section{MATERIAL Y MÉTODOS}

El trabajo se llevó a cabo en el establecimiento "La Pipa", ubicado a 5,6 Km de la localidad de Tartagal, al norte de la provincia de Santa Fe. La empresa se dedica a la cría bovina, con servicio estacionado de primavera, que va desde octubre a enero, y destete tradicional de las crías con 6 meses de edad.

Para el ensayo se utilizaron terneros machos Braford $(n=28)$ que se encontraban al pie de la madre y sobre una base de campo natural pastoreando un recurso forrajero de campo natural. Mediante un diseño experimental en bloques completos al azar, factorial 2 x 2 (dos tratamientos, enteros y castrados y dos edades, 45 y 90 días de vida con una diferencia inferior a una semana), todos hijos de vacas multíparas.

Se asignaron aleatoriamente 7 individuos a cada grupo: castrados a los 45 días de vida (C45); enteros con 45 días de vida (E45); castrados a los 90 días de vida (C90); enteros a los 90 días (E90). Los enteros en ambas edades fueron utilizados como testigos.

Los sujetos de estudio fueron identificados con caravanas tarjeta-botón, sus pesos se registraron matutina e individualmente el día 0 (día de la esterilización) y posteriormente los días 15,30 y 45 , mediante balanza para ganado con capacidad de $3.000 \mathrm{~kg}$. La castración se realizó mediante técnica quirúrgica cruenta, sin mitigante del dolor ${ }^{11,12}$, dado que era el utilizado de rutina por el personal veterinario del establecimiento.

Los animales en estudio se mantuvieron en las mismas condiciones nutricionales sobre pastizal natural. El día cero del ensayo, con el fin de reducir la carga parasitaria, recibieron una dosis de $0,3 \mathrm{mg} / \mathrm{kg}$ de doramectina al 1\% (Dectomax ${ }^{\circledR}$, Pfizer). A los terneros castrados, para prevenir infecciones, también se les aplicó -a dosis única- $20 \mathrm{mg} / \mathrm{kg}$ de oxitetraciclina larga acción(Terramicina ${ }^{\circledR}$ Pfizer).

Las variables estudiadas fueron: ganancia total (GT, expresada en $\mathrm{kg}$ de peso vivo) y ganancia media diaria (GMD, expresada en kg/día). En primera instancia, se realizó el análisis descriptivo de la población, calcu- 
Tabla 1. Estadística descriptiva de las variables ganancia total $(\mathrm{kg})$ y ganancia media diaria ( $\mathrm{kg} / \mathrm{día})$, según edad de castración/testigo.

\begin{tabular}{lccccccc}
\hline variable & edad & $\mathrm{n}$ & media & $\mathrm{DE}( \pm)$ & $\mathrm{CV}(\%)$ & mín & máx \\
\hline ganancia & 45 días & 14 & 36,07 & 10,37 & 28,74 & 16 & 54 \\
total & 90 días & 14 & 47,71 & 8,48 & 17,77 & 32 & 64 \\
\hline ganancia & 45 días & 14 & 0,60 & 0,17 & 28,73 & 0,27 & 0,9 \\
media diaria & 90 días & 14 & 0,80 & 0,14 & 17,79 & 0,53 & 1,07 \\
\hline
\end{tabular}

DE: desvío estándar, CV: coeficiente de variación, mín: mínimo, máx: máximo.

Tabla 2. Ganancia total (GT) y ganancia media diaria (GMD), en grupos de 7 terneros cada uno (test de Duncan, $\alpha=0,05$ ).

\begin{tabular}{llcccc}
\hline variable & & C45 & E45 & C90 & E90 \\
\hline \multirow{2}{*}{ GT } & medias $(\mathrm{kg})$ & $34,86^{\mathrm{a}}$ & $37,29^{\mathrm{a}}$ & $51,14^{\mathrm{b}}$ & $44,29^{\mathrm{ab}}$ \\
& EE $( \pm)$ & 3,57 & 3,57 & 3,57 & 3,57 \\
\hline \multirow{2}{*}{ GMD } & medias (kg/día) & $0,58^{\mathrm{a}}$ & $0,62^{\mathrm{a}}$ & $0,85^{\mathrm{b}}$ & $0,74^{\mathrm{ab}}$ \\
& EE $( \pm)$ & 0,06 & 0,06 & 0,06 & 0,06 \\
\hline
\end{tabular}

C45 y E45: castrados y enteros con 45 días de vida. C90 y E90 castrados y enteros a los 90 días. EE: error estándar. Medias con una letra común no son significativamente diferentes $(\mathrm{p}>0,05)$.

lándose: media aritmética, desvío estándar, máximos, mínimos y coeficientes de variación porcentual.

La distribución de las variables se constató mediante el test de Shapiro-Wilks (modificado) y la homocedasticidad mediante el test de Levene. Posteriormente, se analizaron las variables mediante el análisis de la varianza (ANOVA), tomándose como efecto principal la edad de castración a los 45 y 90 días, con sus respectivos controles (enteros). Las medias ajustadas de mínimos cuadrados se compararon por el test de Duncan $(p<0,05)$. Para analizar los datos se utilizó el software InfoStat-Statistical ${ }^{9}$.

\section{RESULTADOS Y DISCUSIÓN}

En la Tabla 1 se muestran los datos estadísticos descriptivos de las variables ganancia total y diaria de peso de terneros (enteros y castrados) según la edad de castración.

El ANOVA arrojó diferencias significativas para las variables ganancia total $(\mathrm{p}=0,0156)$ y diaria de peso $(p=0,0157)$, siendo superior $\mathrm{C} 90$ en 13,27 y $32 \%$ al relacionar con E90, E45 y C45 respectivamente.

En la Tabla 2 se pueden observar las medias ajustadas por mínimos cuadrados para dichas variables, así como el error estándar y los resultados del test de Duncan. En C45 y E45 no hubo diferencias estadísticas debidas al tratamiento. En un ensayo realizado en General Hornos (Provincia de Buenos Aires), otros autores obtuvieron en terneros Aberdeen Angus resultados superiores a los aquí obtenidos, reportando ganancias totales de 41,04 y $32,58 \mathrm{~kg}$, y ganancias diarias de 0,912 y 0,724

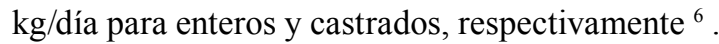

En Chile, en terneros cruza Angus x Hereford, se informaron diferencias significativas a favor de los ejemplares castrados, dato semejante aunque superior- a nuestros resultados, obteniendo ganancias totales de 58,05 y $41,85 \mathrm{~kg}$ y ganancias diarias de $1,29 \mathrm{y}$ $0,93 \mathrm{~kg} / \mathrm{dí}$ en castrados y enteros respectivamente ${ }^{1}$.

También en Chile, en terneros Hereford, contrariamente a los resultados anteriores, se obtuvieron diferencias significativas entre ejemplares castrados a los 90 días y enteros a la misma edad, con valores de 1,028 y $0,908 \mathrm{~kg}$ respectivamente; siendo valores muy superiores a los del presente trabajo ${ }^{16}$.

Se concluye que la castración de los terneros debería realizarse a los tres meses de edad, dado que en ambas variables de crecimiento, los pesos de animales castrados a los 90 días fueron superiores respecto a los terneros de ambos grupos de 45 días de edad.

\section{REFERENCIAS}

1. Arias R, Pérez FJ, Velásquez A, Alvarado AC. 2014. Ganancia de peso diaria de machos castrados vs. machos enteros durante la recría. Agro Sur 42: 23-29.

2. Bretschneider G. 2005. Effects of age and method of castration on performance and stress response of beef male cattle. Livest Sci 97: 89-100.

3. Bretschneider G. 2009. Castración de terneros: tradición versus eficiencia. Redvet 10: 3.

4. Carrillo J. 2017. Manejo de un rodeo de cría, $1^{\circ}$ ed., $14^{\circ}$ reimpresión, Ed. INTA, Buenos Aires, p. 194.

5. Council FAW. 1981. Advice to Agricultural Ministers of Great Britain on the need to control certain mutilations on farm animals, Ed. Middlesex, UK, p. 10.

6. Delaorden J, Demarco D. 2005. Efecto de la edad de castración en la ganancia diaria de peso hasta el destete en terneros al pie de madre. Rev Med Vet 86: 110.

7. Delcampo M. 2013. El bienestar animal aplicado a los sistemas de producción de ganado vacuno. I Simpósio Internacional sobre Sistemas de Produção de Bovinos de Corte, Fac.Agron.Univ.Fed.Rio Grande do Sul, Porto Alegre, Brasil, p. 132-163.

8. Dimarco O. 1998. Crecimiento de vacunos para carne, $1^{\circ}$ ed., INIA, Mar del Plata, Argentina, p. 246.

9. Dirienzo JA et al. 2018. InfoStat versión 2018. Grupo InfoStat. FCA, Univ.Nac. de Córdoba, Argentina, http:// www.infostat.com.ar.

10. Federation of Animal Science Societies (FASS). 2010. Guide for the care and use of agricultural animals in research and teaching, $3^{\circ}$ Ed. Champlain, USA, p. 177.

11. Fubini S, Ducharme N. 2005. Cirugía en bovinos. En: $\mathrm{Ci}$ rugía en animales de granja, $1^{\circ}$ ed., Intermédica, Buenos Aires, Argentina, p. 133-478.

12. Garnero OJ, Perusia OR. 2002. Aparato reproductor del macho. En: Manual de anestesias y cirugías de bovinos, $2^{\circ}$ ed., Imprenta San Cayetano, Esperanza (Santa Fe, Argentina), p. 76-91.

13. Ismail H. 2006. Effect of castration on feedlot performance, carcass characteristics and meat quality of 
Westren Sudan Baggara bulls. MSc Thesis, Faculty of Animal Production, University of Khartoum, Sudan, p. 117.

14. Micol D et al. 2009. Effect of age at castration on animal performance, muscle characteristics and meat quality traits in 26-month-old Charolais steers. Livest Sci. 120: 116-126.

15. Moreira FV et al. 2015. Effects of surgical castration, immunocastration and homeopathy on the performance, carcass characteristics and behaviour of feedlot-finished crossbred bulls. Sem Ciênc Agr 36: 1725-1734.

16. Rojas C. 1986. Efecto de tres niveles de suplementación invernal y tres edades de castración sobre la ganancia de peso y calidad de la canal en toritos Hereford. Agric Tecn Chile 46: 21-26.
17. Sampedro D, Calvi M. 2018. Caracterización de la ganadería vacuna del nordeste argentino. En: Cría vacuna en el NEA (Capitulo 1), Ed. INTA Ediciones, Corrientes, Argentina, p. 9-15.

18. Warnock T et al. 2012. Effect of castration technique on beef calf performance, feed efficiency, and inflammatory response. J Anim Sci 90: 2345-2352.

\section{Asociación Cooperadora de la Facultad de Ciencias Veterinarias Universidad Nacional del Nordeste}

Personería Jurídica No 647/92 y 912/00

Sargento Cabral 2139

3400 Corrientes

Argentina

La Asociación Cooperadora de la Facultad de Ciencias Veterinarias de la UNNE fue constituida el 10 de diciembre del año 1991 como entidad de bien público, con el objeto de promover y coadyuvar las actividades científicas, educativas y culturales relacionadas con las Ciencias Veterinarias. En tal sentido, implementa acciones para colaborar con la enseñanza, extensión, actualización y difusión científica que realiza dicha casa de estudios.

\section{Beneficios que brinda a sus asociados:}

- Fotocopias con descuentos especiales en la Fotocopiadora Copias.com que funciona dentro del predio de la Facultad de Ciencias Veterinarias.

- Descuentos para la adquisición de libros de distintas editoriales.

- Descuentos especiales en otros rubros.

Tel. (0379) 4425753 interno 186•Página Web www.vet.unne.edu.ar•E-mail: cooperadora@vet.unne.edu.ar 